\title{
MCQs in Management and Healthcare Administration
}

1. Planning a new hospital starts with setting goals for the hospital, without which the organization cannot have a definite direction or focus. Which of the following areas is not considered by an organization while studying existing hospital facilities in an area?
(a) Bed ratio
(b) Hospital occupancy
(c) Physical condition of facilities
(d) Volume of services provided
(e) Perception of patients

2. Centralization principle of management science has brought the existence of central sterile services department (CSSD) in large hospitals. The significance of CSSD is:

(a) To bring advancement in medical science to provide betterment of humanity

(b) To help the doctors to develop leadership qualities and human relationship abilities

(c) To bring efficiency and economy in the centralization of sterilization of syringes activity in one location for use all over the hospital

(d) To help nonmedical staff to learn administrative skills

(e) To help medical professionals to develop management attitudes.

3. There are various operational research models used in the areas of hospital and healthcare management. Which of the following models is used in the capital rationing of resources like budget allocation, transfer pricing, etc.?
(a) Assignment model
(b) Transportation model
(c) Dynamic programming model
(d) Sequencing model
(e) Linear programming model.

4. All drugs are classified individually on the basis of A-B-C, X-Y-Z, H-M-L, V-E-D, F-M-S and G-O-F analysis. The set of these six classifications, to which every item belongs, is then defined as a new category in the multiple basis approach to selective inventory control (MBASIC) system called classification-combination. Apart from this classificationcombination, If nonmoving drugs $(\mathrm{N})$ along with the drugs classified on the basis of value, unit price, consumption pattern and sources of supply are considered, the total number of possible classification combinations that can emerge is:
(a) 27
(b) 81
(c) 243
(d) 729
(e) 810 .

5. A medical audit committee comprises professionals from different departments of a hospital. Which of the following may act only as a observer in committee meetings?
(a) Heads of medical departments
(b) Nursing superintendents
(c) Head of pathology
(d) Director of medical services
(e) Administrator.

6. A hospital is a vital organization and stands unique and incomparable to any other organization. Which of the following is considered important part of new hospital plan, not only for aesthetics and access, but also to utilize the potential to capture or avoid natural energy?
(a) Provision for disasters
(b) Functional requirements
(c) Future expansion
(d) Environmental impact analysis
(e) Citing and orientation.

7. Which of the following is determined by calculating deaths after 24 hours of hospital admission?
(a) Gross death rate
(b) Random death rate
(c) On-the-spot death rate
(d) Net death rate
(e) Chronic death rate.

8. Harrison suggested different methods of monitoring of medical audit. Which of the following method of monitoring is based on the primary idea of learning from mistakes?
(a) Peer review
(b) Sentinel cases
(c) Criterion-based audit
(d) Surveys
(e) Comparison of the small groups in the same field.

9. Laboratory and diagnostic services are of paramount importance in a hospital because

(a) The relationship formed at this stage go a long way in building overall image of the hospital

(b) This stage forms the initial contact point between patient and the hospital

(c) Any amount of effort expended at this stage is less, unless one can delight the patient

(d) They properly receive the patients and brief them about the investigations

(e) Their indirect interaction also has a definite bearing on the final outcome of the patients satisfaction.

10. A hospital requires to check and understand the applicability of the standard clauses for the institution. In India, all the hospitals have been certified for
(a) ISO 9000
(b) ISO 9001
(c) ISO 9002
(d) ISO 9003 


\section{Answers of MCQs in Management and Healthcare Administration}

\section{Q. 1. Answer: e}

An organization considers following points while studying existing hospital facilities in an area:

- Bed ratio

- Hospital occupancy

- Physical condition of facilities

- Volume of services provided.

\section{Q. 2. Answer: c}

The significance of Central Sterile Services Department (CSSD) is to bring efficiency and economy in the centralization of sterilization of syringes activity in one location for use all over the hospital. Hence, option (c) is the correct answer.

\section{Q. 3. Answer: e}

Linear programming model is used in capital rationing of resources, budget allocation, transfer pricing, etc. Hence, option (e) is the correct answer.

\section{Q. 4. Answer: e}

All drugs are classified individually on the basis of A-B-C, X-Y-Z, H-M-L, V-E-D, F-M-S and G-O-F analysis. The set of these six classifications, to which every item belongs, is then defined as a new category in the multiple basis approach to selective inventory control (MBASIC) system called classification-combination. The total number of classification combinations that can emerge is sn, where:

$\mathrm{s}=$ the number of categories in each selective control technique

$\mathrm{n}=$ the number of selective control techniques

In the present study, $\mathrm{n}=6$ and $\mathrm{s}=3$

The total number of classification will be $36=729$.
Nonmoving drugs classified on the basis of value, unit price, consumption pattern and sources of supply are classified as four types, such as A-B-C, H-M-L, F-M-S and G-O-F. Each classification is having three categories. The classification-combination will be $(\mathrm{n}=$ $4, s=3)=81$. The total number of possible classification-combination is $729+81=810$.

Hence, option (e) is the correct answer.

\section{Q. 5. Answer: e}

Administrators may act only as a observer in committee meetings, if they do not have a medical background. Hence, option (e) is the correct answer.

\section{Q. 6. Answer: e}

Citing and orientation is considered important part of new hospital plan, not only for aesthetics and access but also to utilize the potential to capture or avoid natural energy. Hence, option (e) is the correct answer.

\section{Q. 7. Answer: d}

Net death rate is determined by calculating deaths after 24 hours of hospital admission. Hence, option (d) is the correct answer.

\section{Q. 8. Answer: a}

Peer review is a method of monitoring based on the primary idea of learning from mistakes. Hence, option (a) is the correct answer.

\section{Q. 9. Answer: d}

Laboratory and diagnostic services are of paramount importance in a hospital because they properly receive the patients and brief them about the investigations. Hence, option (d) is the correct answer.

Q. 10. Answer: c

In India, all the hospitals have been certified for ISO 9002. Hence, option (c) is the correct answer. 